\title{
Assistência à saúde de adolescentes de minorias sexuais e de gênero: scoping review
}

\author{
Health Care for Sexual and Gender Minority Adolescents: scoping review \\ Atención de salud para adolescentes de minorías sexuales y de género: revisión del alcance
}

Recebido: 14/01/2022 | Revisado: 19/01/2022 | Aceito: 26/01/2022 | Publicado: 27/01/2022

\author{
Sabrina Brombim Zanchetta \\ ORCID: https://orcid.org/0000-0002-9505-6757 \\ Universidade Federal de São João del Rei, Brasil \\ E-mail: sabrinabrombim@hotmail.com \\ Edilene Aparecida Araújo da Silveira \\ ORCID: https://orcid.org/0000-0001-7378-2240 \\ Universidade Federal de São João del Rei, Brasil \\ E-mail: edileneap@ufsj.edu.br
}

\begin{abstract}
Resumo
Objetivo: Mapear as evidências cientificas sobre assistência em saúde aos adolescentes de minorias sexuais e de gênero no contexto da atenção primária. Metodologia: Scoping Review, que foi desenvolvida com base nas recomendações do guia internacional Joanna Briggs Institute Reviewers. Foi realizada busca em bases de dados selecionadas que continham artigos na íntegra e que respondessem ao questionamento: quais seriam os conhecimentos ou evidências científicas que poderiam nortear a elaboração de protocolos e ações educativas no contexto da atenção primária em saúde? Foram realizadas buscas em seis bases de dados internacionais e nacionais, sobre trabalhos publicados até 2021. Resultados: Dentre os 394 estudos, 25 foram selecionados para leitura na integra com uma amostra final de 11 estudos. Os 11 estudos foram publicados de 2017 a 2020. Os estudos selecionados são de âmbito internacional e de abordagem quantitativa, qualitativa e qualiquantitativa. Os estudos trouxeram importantes propostas de intervenções que podem ser realizadas para melhorar o atendimento de profissionais no ambiente da atenção primária. Conclusão: Percebe-se que o campo de estudo é pouco explorado pela literatura nacional e os estudos são observacionais, ocorrendo uma lacuna em relação a diversidade de atendimento para minorias sexuais e de gênero que deve ser preenchida por estudos futuros.
\end{abstract}

Palavras-chave: Adolescente; Minorias sexuais e de gênero; Atenção Primária à Saúde.

\begin{abstract}
Objective: To map scientific evidence on health care for adolescents from sexual and gender minorities in the context of primary care. Methodology: Scoping Review, which was developed based on the recommendations of the Joanna Briggs Institute Reviewers international guide. A search was carried out in selected databases that contained full articles and answered the question: what would be the knowledge or scientific evidence that could guide the development of protocols and educational actions in the context of primary health care? Searches were performed in six international and national databases, on works published until 2021. Results: Among the 394 studies, 25 were selected for full reading with a final sample of 11 studies. The 11 studies were published from 2017 to 2020 . The selected studies are of international scope and have a quantitative, qualitative and qualiquantitative approach. The studies brought important proposals for interventions that can be carried out to improve the care provided by professionals in the primary care environment. Conclusion: It is noticed that the field of study is little explored in the national literature and studies are observational, with a gap in relation to the diversity of care for sexual and gender minorities that should be filled by future studies.
\end{abstract}

Keywords: Adolescent; Sexual and gender minorities; Primary Health Care.

\section{Resumen}

Objetivo: Mapear la evidencia científica sobre la atención a la salud de adolescentes de minorías sexuales y de género en el contexto de la atención primaria. Metodología: Scoping Review, que se desarrolló en base a las recomendaciones de la guía internacional Joanna Briggs Institute Reviewers. Se realizó una búsqueda en bases de datos seleccionadas que contenían artículos completos y respondieron a la pregunta: ¿cuál sería el conocimiento o evidencia científica que podría orientar el desarrollo de protocolos y acciones educativas en el contexto de la atención primaria de salud? Se realizaron búsquedas en seis bases de datos internacionales y nacionales, sobre trabajos publicados hasta 2021. Resultados: Entre los 394 estudios, se seleccionaron 25 para lectura completa con una muestra final de 11 estudios. Los 11 estudios fueron publicados entre 2017 y 2020. Los estudios seleccionados son de alcance internacional y tienen un enfoque cuantitativo, cualitativo y cualitativo. Los estudios trajeron importantes propuestas de intervenciones que pueden llevarse a cabo para mejorar la atención brindada por los profesionales en el ámbito de 
la atención primaria. Conclusión: Se advierte que el campo de estudio está poco explorado en la literatura nacional y los estudios son observacionales, con una brecha en relación a la diversidad de atención a las minorías sexuales y de género que debe ser llenado con estudios futuros.

Palabras clave: Adolescente; Minorías sexuales y de género; Atención Primaria de Salud.

\section{Introdução}

A formação de grupos e movimentos sociais que preconizam as minorias de gênero no Brasil avança a partir de 1970, assim como o movimento da reforma sanitária, que luta pela saúde universal e começa a ser entendida de maneira ampliada e não apenas como assistência médica. As práticas dos ativistas possibilitaram importantes mudanças e reflexões sobre as identidades de gênero e as sexualidades, dando visibilidade política aos problemas enfrentados por grupos socialmente excluídos da vida privada e das relações sociais (Brasil, 2012).

Essa imposição cultural acarreta em vivências constantes de discriminação, podendo desencadear nos serviços de saúde condutas de discriminação, que não respeitem a sexualidade ou a identidade de gênero de adolescentes que não se enquadram nas normas heterossexual e identidades de gênero fora do binarismo feminino/masculino (Guimarães et al., 2018).

A Organização Mundial da Saúde (OMS) compreende o período da adolescência entre 10 e 19 anos de idade, quando ocorrem diversas transformações biopsicossociais. Estudos revelam que os jovens passam por descobrimentos de sua sexualidade e identidade de gênero na adolescência, fase essa atravessada por transformações e descobertas que envolvem questões sociais e culturais, culminando na busca de sua identidade. Considerando a sexualidade e a identidade de gênero na adolescência como algo complexo e multifacetado, o adolescente possui pouca maturidade para aceitar sua sexualidade e identidade de gênero, já que está inserido em uma cultura de crenças e tabus heterocisnormativo (Guimarães et a.1, 2018).

Desse modo, esses adolescentes possuem seus direitos violados, podendo enfrentar desafios à saúde e ao seu bemestar, como: bullying, exclusão social e familiar, baixa autoestima, interações negativas no sistema de saúde, maiores riscos de violência e desemprego (Guimarães et al., 2018).

Além disso, jovens que possuem uma orientação sexual ou identidade de gênero diversas não são compreendidos pela heterocisnormatividade estabelecida, passando por inúmeras formas de preconceitos sociais e são invisibilizados socialmente, podendo ocorrer esse processo no âmbito da saúde. Considera-se, a importância dos profissionais de saúde como um apoio e confiabilidade para que o adolescente possa se expressar e entender esses diversos desafios (Guimarães et al., 2018).

Diante disso, as políticas de saúdes devem identificar as necessidades dos grupos vulneráveis, dentre eles adolescentes Lésbicas, Gays, Bissexuais, Transexuais e Travestis (LGBTs) colaborando para reduzir a desigualdade dos determinantes sociais e possibilitar o acesso à assistência integral de saúde. É nesse sentido que a Atenção Primária à Saúde (APS) se torna um importante local de acolhimento desses jovens, já que é a porta de entrada do Sistema Único de Saúde (SUS) e um espaço onde ocorre encontros entre os usuários, estudantes e profissionais da saúde, possibilitando o conhecimento do território, identificando suas necessidades e realizando um trabalho efetivo com a comunidade (Guimarães et al., 2019).

Mesmo com garantias constitucionais desses princípios, os profissionais possuem dificuldades em abordar temáticas relacionadas à saúde e sexualidade com os adolescentes LGBTs. As temáticas relacionadas à sexualidade e gênero não integram o currículo de universidades e cursos preparatórios de profissionais (Guimarães et al., 2020). O déficit na formação e ausência de cursos que abordem o assunto direcionados a profissionais podem culminar em processo de exclusão nos serviços de saúde más condutas de profissionais sob a forma de atitudes preconceituosas, atendimentos não humanizados, dificuldade de compreensão sobre a complexidade do atendimento (Belém et al., 2018).

As atitudes que violam direitos humanos, reduzem a acessibilidade aos serviços de saúde e aumentam a exposição dos adolescentes aos agravos em saúde. As desigualdades geram a invisibilidade da saúde dos adolescentes de minorias de gênero, causando a falta de diretrizes e de protocolos específicos para esse público (Belém et al., 2018). 
Ademais, como mencionado, os profissionais possuem uma formação que não prioriza o atendimento em saúde a essa população, fazendo com que a assistência se torne desqualificada ao assistir os adolescentes. Protocolos, educação continuada e outras estratégias poderiam trazer conhecimentos que norteassem a assistência em saúde. Assim, questiona-se: quais seriam os conhecimentos ou evidências científicas que poderiam nortear a elaboração de protocolos e ações educativas no contexto da atenção primária em saúde?

A política nacional de saúde integral de lésbicas, gays bissexuais, travestis e transexuais recomenda, a partir da $13^{\circ}$ Conferência Nacional de Saúde, "A sensibilização dos profissionais a respeito dos direitos LGBTs, com inclusão do tema livre expressão sexual na política de educação permanente no SUS" (Brasil, 2012, p. 12); e "O estabelecimento de normas e protocolos de atendimento específicos para as lésbicas e travestis" (Brasil, 2012, p.12).

Considerando os desafios a serem enfrentados pelos adolescentes LGBTs e a necessidade de um olhar crítico dos profissionais sobre determinantes em saúde específicos, o objetivo desse estudo é mapear as evidências cientificas sobre assistência em saúde aos adolescentes de minorias sexuais e de gênero no contexto da atenção primária. Após essa síntese, descreveremos o processo de construção de um protocolo para atendimento dessa população.

\section{Metodologia}

O presente estudo é uma Scooping review, que foi desenvolvida com base nas recomendações do guia internacional Joanna Briggs Institute Reviewers Manual 2020 e apresentada conforme o PRISMA Extension for Scoping Reviews (PRISMAScR): Checklist and explanation (Tricco, et al., 2021; Peters, et al., 2018). A scoping review foi registrada na plataforma Open Science Framework (OSF) e encontra-se disponível no doi: 10.17605/osf.io/byjud.

Foram seguidas as seguintes etapas: 1) identificação da questão de pesquisa, 2) identificação dos estudos relevantes; 3) seleção dos estudos; 4) análise dos dados; 5) agrupamento, síntese e apresentação dos dados (Tricco, et al., 2021). Essa revisão pode ser utilizada como ferramenta de mapeamento do conceito chave proposto pela pesquisa, esclarecendo o que foi proposto (Tricco, et al., 2021). Tem a possibilidade de realizar uma análise ampla de literatura e apresentar publicações que discutam atuação dos profissionais na atenção primária a adolescentes de minorias sexuais e de gênero para que seja discutido melhores atuações em saúde.

A questão da pesquisa, o objetivo do estudo e os descritores foram definidos de acordo com a estratégia participantes, conceito e contexto (PCC) onde P significa participantes (Adolescente LGBT), C é conceito (Minorias sexual e de gênero) e C é contexto (Atenção primária à saúde).

A princípio foi realizada uma pesquisa no portal PubMed para identificar os principais descritores e palavras-chaves utilizados nos estudos da temática apresentada a partir da combinação dos MeSH’s identificados: (c(c((("Primary Health Care"[Mesh]) AND "Sexual and Gender Minorities"[Mesh]) AND ("Transgender Persons"[Mesh] OR "Health Services for Transgender Persons"[Mesh])) AND "Health Equity"[Mesh]) OR "Universal Health Care"[Mesh]) OR "Right to Health"[Mesh]. Diante disso, a estratégia de busca final foi definida por: (("Adolescent"[Mesh]) AND "Primary Health Care"[Mesh]) AND "Sexual and Gender Minorities"[Mesh].

A busca foi realizada no período de 12 a 25 de julho de 2021, nas seguintes bases de dados: Medline via PUBMED (National Library of Medicine and National Institutes of Health), CINAHL (Cummulative Index to Nursingand Allied Health Literature), Web of Science, SCOPUS, LILACS (Literatura Latino-Americana e do Caribe em Ciências da Saúde) e biblioteca Cochrane Central. Em todas essas a pesquisa aconteceu através da busca avançada e de forma controlada. Um outro recurso empregado, quando possível, foi o uso de filtros de coleta. 
Os artigos foram acessados via Portal de Periódicos da Coordenação de Aperfeiçoamento de Pessoal do Nível Superior (CAPES), através da Comunidade Acadêmica Federada (CAFe), com acesso da instituição de ensino superior Universidade Federal de São João Del Rei - CCO - Dona Lindu (UFSJ).

Foram considerados os seguintes critérios de inclusão: artigos publicados em português, inglês ou espanhol, com resumos disponíveis na íntegra nas bases de dados selecionadas, que abordem atendimento na atenção primária a adolescentes de minorias sexuais e de gênero, além de que, não houve limitação de período de publicação dos artigos. Foram excluídos da seleção artigos que não contemplem a pergunta, artigos que não apresentam o resumo nas bases de dados e não disponíveis no formato de texto completo, artigos escritos em idioma que não definidos no estudo, resumos e anais de congressos, comentários, editoriais, opiniões, notas prévias, relatórios, artigos de revisão narrativa e de revisão crítica. Para compor os materiais de literatura cinzenta foram utilizadas as bases de dados do Ministério da Saúde.

Após a identificação dos artigos recuperados na busca, houve a leitura e análise de resumos e títulos por dois revisores, que trabalharam independentemente para identificar aqueles que foram considerados potencialmente elegíveis para o estudo. Em casos de dúvidas, os artigos foram elencados para a fase seguinte. Nessa etapa os dois revisores liam os artigos na íntegra com a finalidade de confirmar a pertinência da pergunta de revisão e, em caso positivo, extrair os dados de interesse. As dúvidas foram resolvidas em consenso entre os autores.

$\mathrm{Na}$ etapa de separação, sumarização e relatório dos elementos essenciais encontrados em cada estudo, foi utilizado um instrumento estruturado adaptado do formulário recomendado pelo JBI (Tricco, et al., 2021). Esse instrumento permitiu a síntese, a interpretação de dados e a análise numérica básica, natureza e distribuição dos estudos incorporados na revisão. Foram coletados os dados relacionados a título, autores, ano de publicação, periódico, país de publicação, método (abordagem), nível de evidência, intervenções recomendadas e principais conclusões que respondiam à pergunta norteadora.

Assim, em cada publicação foram identificadas e extraídas as partes fundamentais envolvidas na conjectura do problema, nos contextos, nos métodos, nas discussões e conclusões. Os núcleos fundamentais foram analisados e identificadas as categorias de análise que permitiram sintetizar de forma narrativa os achados. $\mathrm{O}$ artigo era retomado sempre que houvesse alguma necessidade durante essa fase de análise. A estatística descritiva foi utilizada para a análise do material.

\section{Resultados}

A busca inicial nas bases de dados gerou um total de 394 artigos para a análise de título. Na primeira fase, após exame do título e resumo (sempre que necessário), foram pré-selecionados 68 estudos, destes 320 foram excluídos segundo os critérios de exclusão após a leitura na íntegra. Assim, a amostra deste estudo foi constituída por 11 estudos. A Figura 1, mostra o processo de busca, de exclusão e seleção dos artigos. 
Figura 1 - Fluxo referente ao processo de seleção dos estudos da scoping review, 2021.

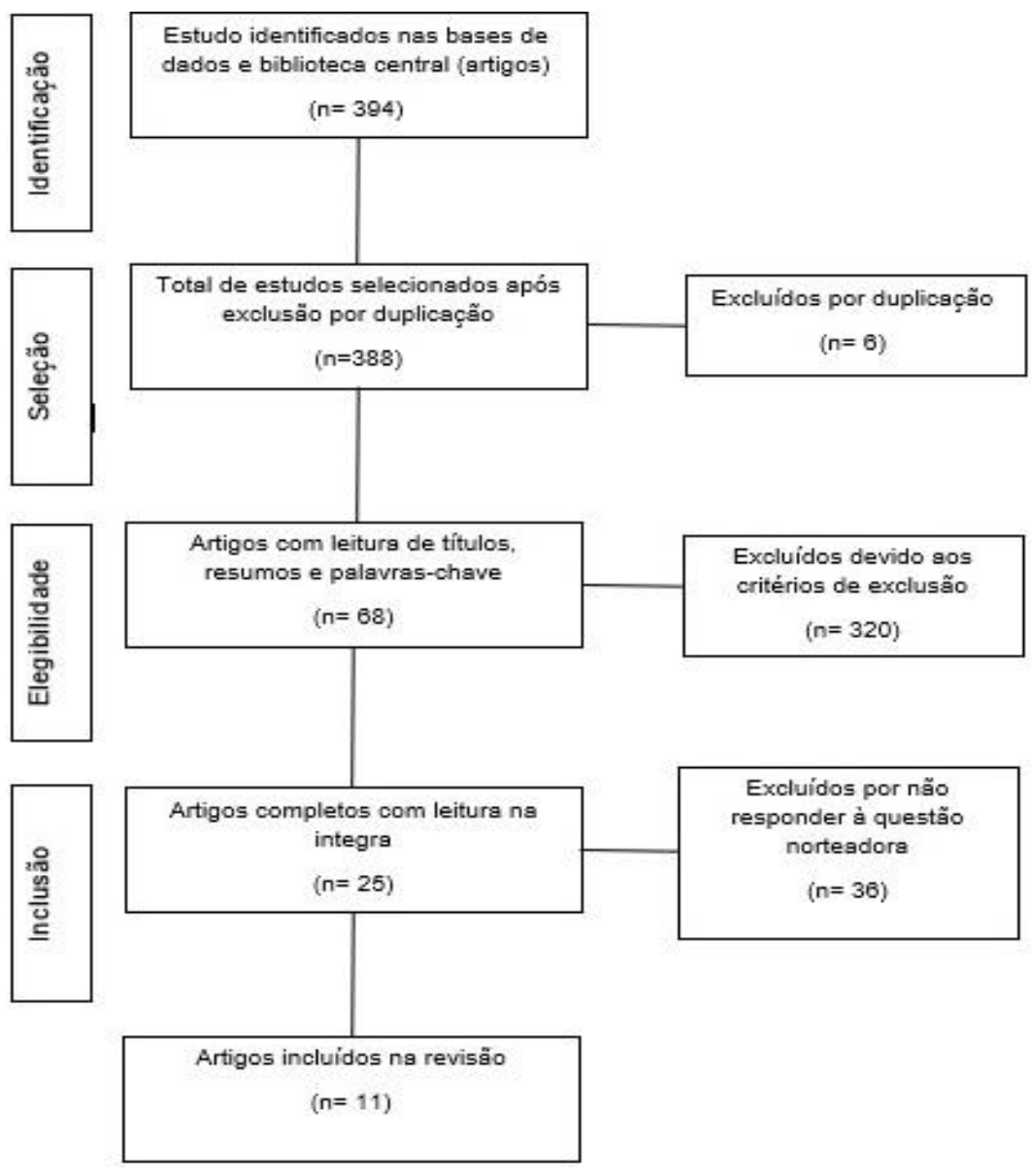

Fonte: Minas Gerais, Brasil (2021).

Os artigos analisados foram organizados no Quadro 1, a seguir e, evidencia os seguintes dados: autores, ano de publicação, revista publicada, país onde foi realizado o estudo, nível de evidência, principais resultados que respondem à pergunta norteadora, abordagem metodológica.

Quadro 1 - Distribuição dos artigos analisados de acordo com ano de publicação, título, pais, desfechos, autores, intervenções e nível de evidencia.

\begin{tabular}{|c|c|c|c|c|c|}
\hline Referência (s) & $\begin{array}{l}\text { Ano } \\
\text { País }\end{array}$ & $\begin{array}{l}\text { Delineamento/nú } \\
\text { mero de } \\
\text { pacientes }\end{array}$ & $\begin{array}{l}\text { Intervençõe } \\
\text { s }\end{array}$ & $\begin{array}{l}\text { Desfechos (resultados que respondem à } \\
\text { pergunta norteadora) }\end{array}$ & $\begin{array}{l}\text { Nível de } \\
\text { evidência }\end{array}$ \\
\hline $\begin{array}{l}\text { Goldenberg, T. et al. Stigma, } \\
\text { Gender Affirmation, and } \\
\text { Primary Healthcare Use } \\
\text { Among Black Transgender } \\
\text { Youth. } \\
\text { J Adolesc Health. }\end{array}$ & $\begin{array}{l}2019, \\
\text { Estados } \\
\text { Unidos }\end{array}$ & $\begin{array}{l}\text { Estudo } \quad \text { de } \\
\text { métodos mistos } \\
110 \text { transgêneros } \\
\text { negros e jovens } \\
\text { com diversidade } \\
\text { de renero } \\
(\text { TGDY) negros } \\
\text { de } 14 \text { cidades dos } \\
\text { EUA }\end{array}$ & $\begin{array}{l}\text { Questionário } \\
\text { online. }\end{array}$ & $\begin{array}{l}\text { A abordagem intersetorial a ser usada por } \\
\text { profissionais de saúde, ambientes e políticas } \\
\text { públicas pode aumentar o acesso: à saúde de } \\
\text { transgêneros negros. É necessário treinar os } \\
\text { profissionais de saúde. A afirmação de gênero na } \\
\text { área da saúde atenua a influência do estigma no uso } \\
\text { do serviço de saúde. }\end{array}$ & 6 \\
\hline $\begin{array}{l}\text { Guss, C.E. et al. Transgender } \\
\text { Adolescents' Experiences in } \\
\text { Primary Care: A Qualitative } \\
\text { Study. } \\
\text { J Adolesc Health. }\end{array}$ & $\begin{array}{l}2019, \\
\text { Estados } \\
\text { Unidos }\end{array}$ & $\begin{array}{l}\text { Pesquisa } \\
\text { qualitativa } \\
20 \text { adolescentes } \\
\text { TGN com idades } \\
\text { entre 13-21 anos. }\end{array}$ & $\begin{array}{l}\text { Entrevista } \\
\text { semi } \\
\text { estruturada e } \\
\text { questionário } \\
\text { demográfico }\end{array}$ & $\begin{array}{l}\text { Os adolescentes do estudo fizeram as } \\
\text { recomendações para um ambiente mais acolhedor: } \\
\text { Se referir aos adolescentes com o pronome correto; } \\
\text { panfletos e informações sobre diversidade, saber } \\
\text { informações sobre sua saúde; um banheiro neutro; } \\
\text { adicionar uma pergunta sobre identidade de gênero } \\
\text { e / ou pronomes nos formulários de admissão em } \\
\text { consultórios médicos; manter a privacidade dos } \\
\text { adolescentes; discrição no exame das regiões } \\
\text { genitais e seu tórax; treinar os profissionais. }\end{array}$ & 6 \\
\hline
\end{tabular}




\begin{tabular}{|c|c|c|c|c|c|}
\hline $\begin{array}{l}\text { Taliaferro, L.A. et al. Social } \\
\text { Connectedness Factors that } \\
\text { Facilitate Use of Healthcare } \\
\text { Services: Comparison of } \\
\text { Transgender and Gender } \\
\text { Nonconforming and } \\
\text { Cisgender Adolescents. } \\
\text { JPediatr. }\end{array}$ & $\begin{array}{l}2019, \\
\text { Estados } \\
\text { Unidos }\end{array}$ & $\begin{array}{l}\text { Estudo caso } \\
\text { controle } \\
\text { Estudantes de } \\
\text { escolas públicas. }\end{array}$ & Questionário & $\begin{array}{l}\text { Para promover a saúde dos jovens do TGNC, os } \\
\text { médicos devem compreender os fatores distintos } \\
\text { associados à obtenção de cuidados de saúde entre } \\
\text { essa população, como a necessidade de esforços } \\
\text { personalizados focados no fortalecimento da } \\
\text { conexão entre os jovens do TGNC e seus pais para } \\
\text { facilitar o recebimento dos cuidados necessários. }\end{array}$ & 4 \\
\hline 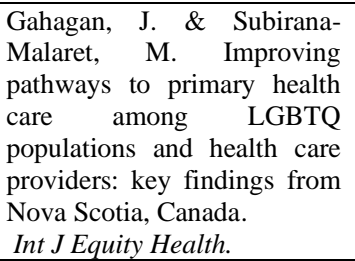 & $\begin{array}{l}\text { 2018, } \\
\text { Canadá }\end{array}$ & $\begin{array}{l}283 \text { adolescentes } \\
\text { LGBTQs maiores } \\
\text { de } 16 \text { anos e } 109 \\
\text { profissionais de } \\
\text { saúde }\end{array}$ & $\begin{array}{l}\text { Entrevistas } \\
\text { online }\end{array}$ & $\begin{array}{l}\text { O atendimento da atenção primária deve melhorar } \\
\text { de forma a ser focado na equidade em saúde, } \\
\text { inclusão, acolhimento e ser culturalmente } \\
\text { competente. Há necessidade de treinamento } \\
\text { adicional e suporte relacionado para profissionais } \\
\text { de saúde que trabalham com as populações } \\
\text { LGBTQ. }\end{array}$ & 6 \\
\hline $\begin{array}{l}\text { Clark, B.A. et al. Primary } \\
\text { care access and foregone } \\
\text { care: a survey of transgender } \\
\text { adolescents and young adults. } \\
\text { Family Practice }\end{array}$ & $\begin{array}{l}\text { 2018, } \\
\text { Canadá }\end{array}$ & $\begin{array}{l}\text { Estudo quanti } \\
\text { qualitativo, } \\
\text { transversal. } 923 \\
\text { Jovens, } \quad 323 \\
\text { adolescentes de } 14 \\
\text { a } 18 \text { anos e } 600 \\
\text { jovens adultos de } \\
19 \text { a } 25 \text { anos. }\end{array}$ & Questionário & $\begin{array}{l}\text { Capacitação para os profissionais; soluções de } \\
\text { políticas, Melhorias no treinamento clínico e nas } \\
\text { ferramentas de prática clínica podem qualificar a } \\
\text { assistência. Tecnologias como o telessaúde } \\
\text { impactam na acessibilidade. A falta de cuidados } \\
\text { necessários varia de barreiras de custos a } \\
\text { experiências negativas anteriores com prestadores } \\
\text { de cuidados de saúde e preocupações sobre a } \\
\text { capacitação de médico. }\end{array}$ & 6 \\
\hline $\begin{array}{l}\text { Fisher, C.B. et al. Perceived } \\
\text { Barriers to HIV Prevention } \\
\text { Services for Transgender } \\
\text { Youth. } \\
\text { LGBT Health. }\end{array}$ & $\begin{array}{l}2018, \\
\text { Estados } \\
\text { Unidos }\end{array}$ & $\begin{array}{l}\text { Estudo } \\
\text { transversal. } \\
\text { Jovens de } 14 \text { a } 21 \\
\text { anos }\end{array}$ & $\begin{array}{l}\text { Questionário } \\
\text { online }\end{array}$ & $\begin{array}{l}\text { Preocupações com estigma e confidencialidade } \\
\text { influenciam negativamente na comunicação dos } \\
\text { jovens transgêneros com os profissionais de saúde. } \\
\text { Destaca-se a importância da privacidade e } \\
\begin{array}{l}\text { confidencialidade, bem como o treinamento de } \\
\text { profissionais sobre a temática. }\end{array}\end{array}$ & 6 \\
\hline $\begin{array}{l}\text { Cafferty, R. et al. Adolescent } \\
\text { Pride Festival Attendees- } \\
\text { Assessing Their Interactions } \\
\text { With Primary Care } \\
\text { Physicians. } \\
\text { J Adolesc Health }\end{array}$ & $\begin{array}{l}2020, \\
\text { Estados } \\
\text { Unidos }\end{array}$ & $\begin{array}{l}\text { Estudo } \\
\text { transversal.534 } \\
\text { adolescentes de } 13 \\
\text { a } 17 \text { anos }\end{array}$ & Questionário & $\begin{array}{l}\text { Os festivais do Orgulho podem ser um local de } \\
\text { acesso à educação e saúde para jovens LGBT. } \\
\text { Especificamente, os festivais do Orgulho podem ser } \\
\text { um local útil para a triagem de DST.Obter um } \\
\text { histórico sexual e rastrear DSTs é algo que todos os } \\
\text { médicos podem fazer e representa um primeiro } \\
\text { passo na iniciativa de redução de DSTs. }\end{array}$ & 6 \\
\hline $\begin{array}{l}\text { Van Donge, N. et al. } \\
\text { Transgender Dependent } \\
\text { Adolescents in the U.S. } \\
\text { Military Health Care System: } \\
\text { Demographics, Treatments } \\
\text { Sought, and Health Care } \\
\text { Service Utilization. } \\
\text { Mil Med. }\end{array}$ & $\begin{array}{l}2019, \\
\text { Estados } \\
\text { Unidos }\end{array}$ & $\begin{array}{l}\text { Estudo descritivo } \\
\text { Prontuários } \quad \text { de } \\
\text { pessoas com } \\
\text { idades entre } \\
\text { anos } 24\end{array}$ & $\begin{array}{l}\text { Revisão } \\
\text { retrospectiva } \\
\text { de } \\
\text { prontuários. } \\
\text { Questionário }\end{array}$ & $\begin{array}{l}\text { Os Jovens TGD podem buscar por atendimento em } \\
\text { várias clínicas. Portanto, a equipe deve ter } \\
\text { sensibilidade, experiência e conhecer a rede de } \\
\text { saúde. O treinamento de profissionais deve } \\
\text { envolver a avaliação com sensibilidade à identidade } \\
\text { e expressão de gênero de seus pacientes. }\end{array}$ & 6 \\
\hline $\begin{array}{l}\text { Luk, J.W. et al. Sexual } \\
\text { attraction and experiences in } \\
\text { the primary care setting: } \\
\text { Examining disparities in } \\
\text { satisfaction with provider and } \\
\text { health self-efficacy. } \\
\text { J Adolesc. }\end{array}$ & $\begin{array}{l}2020, \\
\text { Estados } \\
\text { Unidos. }\end{array}$ & $\begin{array}{l}\text { Ensaio Clínico } \\
\text { Randomizado } \\
535 \text { adolescentes. }\end{array}$ & Questionário & $\begin{array}{l}\text { Os prestadores de cuidados de saúde primários } \\
\text { oferecerem apoio emocional e prático aos jovens de } \\
\text { minorias sexuais para facilitar o estabelecimento de } \\
\text { metas e a realização deles. }\end{array}$ & 2 \\
\hline $\begin{array}{l}\text { Snyder, B.K. et al. LGBTQ } \\
\text { Youth's Perceptions of } \\
\text { Primary Care. } \\
\text { Clin Pediatr }(\text { Phila). }\end{array}$ & $\begin{array}{l}2017, \\
\text { Estados } \\
\text { Unidos. }\end{array}$ & $\begin{array}{l}\text { Estudo } \\
\text { quantiqualitativo } \\
60 \text { jovens. }\end{array}$ & $\begin{array}{l}\text { Grupo focal } \\
\text { Questionário }\end{array}$ & $\begin{array}{l}\text { Os provedores de atenção primária à saúde não } \\
\text { perguntam sobre comportamento sexual e } \\
\text { orientação sexual de seus pacientes pediátricos. Por } \\
\text { outro lado, poucos jovens LGBTQ iniciam } \\
\text { discussões sobre sua orientação sexual com seus } \\
\text { médicos. O ambiente do serviço de saúde precisa } \\
\text { ser mais acolhedor, garantir o sigilo, } \\
\text { confidencialidade e respeito pelos jovens, } \\
\text { considerar as questões de saúde e psicossociais que } \\
\text { afetam LGBTQ. }\end{array}$ & 6 \\
\hline $\begin{array}{l}\text { Guss, C.E. et al. "It'd Be } \\
\text { Great to Have the Options } \\
\text { There": A Mixed-Methods } \\
\text { Study of Gender Identity } \\
\text { Questions on Clinic Forms in } \\
\text { a Primary Care Setting. } \\
\text { J Adolesc Health. }\end{array}$ & $\begin{array}{l}2020, \\
\text { Estados } \\
\text { Unidos }\end{array}$ & $\begin{array}{l}\text { Estudo transversal } \\
21 \text { adolescentes } \\
\text { transgênero, e } \\
\text { cisgênero. }\end{array}$ & $\begin{array}{l}\text { Revisão de } \\
\text { prontuários } \\
\text { Entrevistas } \\
\text { semiestrutur } \\
\text { adas }\end{array}$ & $\begin{array}{l}\text { Inclusão de questões de gênero em formulários } \\
\text { clínicos em ambientes pediátricos; uso de questões } \\
\text { inclusivas e afastamento do foco em identidades } \\
\text { binárias; utilização correta do nome social pelos } \\
\text { profissionais; saber a diferença entre identidade de } \\
\text { gênero e sexualidade. Os participantes descreveram } \\
\text { as perguntas como benéficas para todos os } \\
\text { pacientes e as perceberam como um indicador de } \\
\text { um ambiente clínico acolhedor. Houve aumento } \\
\text { significativo da documentação inclusiva. }\end{array}$ & 6 \\
\hline
\end{tabular}


Fizeram parte da revisão 11 estudos. Os artigos foram escritos em inglês (11). Os trabalhos que deram origem aos artigos foram desenvolvidos em Atenção Primária (7); Redes de atenção (2); Festival do Orgulho (1); Escola (1). Os estudos sobre a temática abrangeram a abordagem quantitativa, qualitativa ou qualiquantitativos, e com objetos de estudo semelhantes sobre o atendimento a adolescentes de minorias sexuais e de gênero e melhorias. A maioria dos artigos foram publicados em 2019 (4), 2020 (3) e 2018 (3). Foram publicados artigos em 2020 (3), 2019 (4), 2018 (3), 2017 (1).

Os trabalhos incluídos na busca foram classificados de acordo com o seu nível de evidência conforme classificação de Melnyk e Fineout-Overholt (2005), ou seja nível 1 (Evidências provenientes de revisão sistemática ou meta-análise de todos relevantes ensaios clínicos randomizados controlados ou provenientes de diretrizes clínicas baseadas em revisões sistemáticas de ensaios clínicos randomizados), nível 2 (Evidências derivadas de pelo menos um ensaio clínico randomizado controlado bem delineado), nível 3 (Evidências obtidas de ensaios clínicos bem delineados sem randomização), nível 4 (Evidência provenientes de estudos de coorte e de caso controle bem delineados), nível 5 (Evidências provenientes de revisão sistemática de estudos descritivos e qualitativos), nível 6 (Evidências derivadas de um único estudo descritivo ou qualitativo) e nível 7 (Evidências provenientes de opinião de autoridades e/ou relatório de especialista).

Os artigos tiveram como nacionalidades, os Estados Unidos (9), Canadá (2). A maioria (9) dos artigos tiveram nível de evidencia 6. O quadro abaixo apresenta todas as intervenções encontradas nos estudos:

Quadro 2 - Distribuição das intervenções indicadas pela literatura de acordo com os autores dos estudos:

\begin{tabular}{|c|c|c|}
\hline Área & Intervenção & Autores \\
\hline \multirow[t]{4}{*}{ Abordagem profissional } & $\begin{array}{l}\text { Uso de linguagem inclusiva, culturalmente aceita e } \\
\text { que estabeleça uma relação de confiança }\end{array}$ & $\begin{array}{l}\text { Goldenberg, et al., 2019; Hadland, et al., 2016; Kano, } \\
\text { et al., } 2016\end{array}$ \\
\hline & $\begin{array}{l}\text { Cuidado que afirme as questões de gênero e sejam } \\
\text { amigáveis }\end{array}$ & Goldenberg, et al., 2019 \\
\hline & $\begin{array}{l}\text { Uso dos pronomes e nome preferencial ao } \\
\text { conversar com o adolescente }\end{array}$ & $\begin{array}{l}\text { Guss, et al., 2019; Hadland, et al., 2016; Jadwin- } \\
\text { Cakmak, 2020; Sefolosha, et al., 2021; Guss, et al., } \\
2020\end{array}$ \\
\hline & Manter a confidencialidade e sigilo & $\begin{array}{l}\text { Guss, et al., 2019; Hadland, et al., 2016; Fisher, et al., } \\
\text { 2018; Sefolosha, et al., 2021; Weiselberg, et al., 2019; } \\
\text { Mayer, et al.,2014; Snyder, et al., } 2017\end{array}$ \\
\hline \multirow[t]{2}{*}{ Ambiente } & $\begin{array}{l}\text { Ambientes seguros, inclusivos, afirmativos } \mathrm{e} \\
\text { acolhedores para atendimento }\end{array}$ & $\begin{array}{l}\text { Goldenberg, et al., 2019; Guss, et al., 2019; Taliaferro, } \\
\text { et al., 2019; Snyder, et al., } 2017\end{array}$ \\
\hline & $\begin{array}{l}\text { Banheiro neutro ou escolha de banheiro de acordo } \\
\text { com o gênero }\end{array}$ & Guss, et al., 2019; Hadland, et al., 2016 \\
\hline \multirow[t]{9}{*}{ Atendimento } & Uso de materiais educativos & Guss, et al., 2019 \\
\hline & $\begin{array}{l}\text { Treinamento de profissionais sobre questões de } \\
\text { gênero, sexualidade e diversidades nos espaços }\end{array}$ & $\begin{array}{l}\text { Goldenberg, et al., 2019; Guss, et al., 2019; Taliaferro, } \\
\text { et al., 2019; Gahagan, et al., 2018; Clark, et al., 2018; } \\
\text { Fisher, et al., 2018; Van Donge, et al., } 2019\end{array}$ \\
\hline & $\begin{array}{l}\text { Incluir questões sobre identidade de gênero na } \\
\text { anamnese e formulários clínicos }\end{array}$ & $\begin{array}{l}\text { Guss, et al., 2019; Taliaferro, et al., 2019; Clark, et al., } \\
\text { 2018; Jadwin-Cakmak, et al., 2020; Guss, et al., } 2020\end{array}$ \\
\hline & Discrição no exame físico da genitália e tórax & Guss, et al., 2019 \\
\hline & Plano de cuidados elaborado junto com o paciente & Wahlen, et al., 2020 \\
\hline & $\begin{array}{l}\text { Remover barreiras ao atendimento e acesso ao } \\
\text { serviço pelos adolescentes }\end{array}$ & Taliaferro, et al., 2019; Mayer, et al., 2014 \\
\hline & $\begin{array}{l}\text { Uso de tecnologias para melhorar a acessibilidade } \\
\text { como o telessaúde e videoconferência }\end{array}$ & Clark, et al., 2018; Kano, et al., 2016 \\
\hline & $\begin{array}{l}\text { Implementar estratégias de promoção e prevenção } \\
\text { em saúde }\end{array}$ & Guimarães, et al., 2019 \\
\hline & Políticas que melhorem a acessibilidade & Clark, et al., 2018 \\
\hline \multirow[t]{3}{*}{ Família e rede social } & $\begin{array}{l}\text { Estimular o relacionamento do adolescente com a } \\
\text { rede social }\end{array}$ & Taliaferro, et al., 2019 \\
\hline & $\begin{array}{l}\text { Educação em saúde sobre a temática para que } \\
\text { pais/familiares discutam identidade de gênero com } \\
\text { os filhos }\end{array}$ & Taliaferro, et al., 2019 \\
\hline & Apoio aos familiares & Van Donge, et al., 2019; Taliaferro, et al., 2019 \\
\hline
\end{tabular}

Fonte: Minas Gerais, Brasil (2021). 
A maioria das intervenções descritas foram relacionadas ao atendimento e à abordagem profissional. Há autores que propuseram intervenções em diferentes áreas.

\section{Discussão}

A atenção primária se destaca por ser a porta de entrada para os jovens, por outro lado, situações de estigmas e exclusão nas instituições podem fazer com que essa população não recorra aos cuidados em saúde, impactando no bem estar ao longo da vida. $\mathrm{O}$ atendimento da atenção primária deve ser focado na equidade em saúde, inclusão, acolhimento e ser culturalmente competente (Gahagan \& Subirana-Malaret, 2018). Por isso, é importante ter em mente que os serviços devem se organizar de acordo com as necessidades dos usuários considerando sua integralidade e construindo modelos de saúde que aumente a participação dos usuários (Gahagan \& Subirana-Malaret, 2018). Neste sentido, os estudos trouxeram várias intervenções a serem realizadas para melhorar o acesso e o ambiente do serviço de saúde.

Os ambientes devem ser atrativos e receptivos em relação a diversidade sexual e a identidade de gênero. $\mathrm{O}$ uso de panfletos direcionado a esse público, linguagens inclusivas, materiais educativos disponíveis, utilização do nome social nos prontuários, banheiros neutros, inclusão de pergunta sobre gênero e/ou pronome em formulários de admissão médica e relações com a rede intersetorial para atendimento em serviços socioassistenciais, apoio jurídico, acesso ao emprego, discussões com a escola, entre outros serviços (Guss, et al., 2019; Goldemberg, et al., 2018; House, et al., 2019; Lait, et al., 2019; Snyder, et al., 2017; Guss, et al., 2020).

Enfim é importante que as clínicas de saúde e os sistemas de atendimento se esforcem para garantir um ambiente que reflita o desejo de inclusão e removam as barreiras que impedem os adolescentes transgêneros de obter os cuidados necessários (Taliaferro, et al., 2019; Kidd, et al., 2020; Mayer, et al., 2014). Alguns artigos discutem que a rede de saúde precisa possuir locais especializados no atendimento à população LGBT, porém há adolescentes que relataram medo de serem alvo de estigma e preconceito ao procurarem pelos serviços com essas características (Kimberly, et al., 2018; Lait, et al., 2019), trazendo dificuldades à acessibilidade e disponibilidade aos cuidados de saúde nesses serviços.

A acessibilidade e disponibilidade dos cuidados em saúde são obstáculos de difícil superação para estes adolescentes, principalmente, quando moram em zonas rurais. Há frequentes relatos de experiências negativas como encontros desconfortáveis e frustrantes com profissionais não familiarizados com cuidados adequados para transgêneros, longos tempos de espera para obter a consulta, falta de conforto e confiança (Clark, et al., 2018).

Estudo encontrou que muitos profissionais, principalmente aqueles que não são LGBT, sentem-se desconfortáveis prestar assistência à essa população (Gahagan \& Subirana-Malaret, 2018). Eles não perguntam rotineiramente sobre comportamento sexual e orientação sexual de seus pacientes e gastam pouco tempo abordando questões que envolvem a sexualidade durante a consulta. Outro problema é que possíveis necessidades de saúde relacionada à população LGBT identificadas pelos profissionais não coincidem com aquelas que o público alvo indica (Gahagan \& Subirana-Malaret, 2018; Snyder, et al., 2017). Portanto há falta de conhecimento sobre as problemáticas vivenciadas por esse público, dificuldade em dialogar sobre sexualidade e identidade de gênero, além dos serviços de saúde não proporcionarem capacitações acerca da temática (Scott, et al., 2017).

Diante desses problemas, os profissionais reconhecem a necessidade de treinamento pois percebem que há um despreparo (Gahagan \& Subirana-Malaret, 2018). O treinamento dos profissionais pode solucionar problemas como a diminuição da disponibilidade, adequação, acessibilidade e aceitabilidade dos cuidados de saúde que adolescentes LGBT tentam acessar (Clark, et al., 2018; Fisher, et al., 2018). Os profissionais precisam de treinamento para compreensão das necessidades e experiências de saúde dessa população, como forma de reduzir as iniquidades e otimizar o cuidado (Goldenberg, et al., 2019; Van Donge, et al., 2019; House, et al., 2019; Mayer, et al., 2014). 
O treinamento pode conter temas relacionados ao fornecimento de cuidados de afirmação de gênero, reconhecimento das potencialidades da rede para o atendimento dessa população, a avaliação da expressão e identidade de gênero dos pacientes, questões de orientação sexual em evolução, comportamento sexual, desenvolvimento psicossocial durante a adolescência no contexto da incongruência de gênero, possíveis causas de experiências negativas de cuidados de saúde para transgêneros e assuntos sobre assistência em saúde que considera gênero na atenção primária (Kidd, et al., 2020; Van Donge, et al., 2019). Entretanto, é útil avaliar as necessidades de educação da equipe com uma ferramenta de avaliação de necessidades para identificar e direcionar as áreas de aprendizagem, uma vez que muitas escolas de formação não possuem esse conteúdo em seus currículos (House, et al., 2019).

Além o treinamento, a abordagem intersetorial e o uso de tecnologias tipo telessaúde podem auxiliar na redução dos obstáculos encontrados pelos adolescentes na disponibilidade e acesso aos cuidados à saúde (Goldenberg, et al., 2019; Clark, et al., 2018). Dentre essas dificuldades estão o estigma e preconceito (Clark, et al., 2018). O estigma pode desestimular o jovem a procurar o serviço de saúde. O estigma e preocupações com a confidencialidade influenciam negativamente na comunicação com o provedor de saúde (Goldenberg, et al., 2019).

Problemas referentes ao sigilo, privacidade e confidencialidade geram inseguranças pois há casos em que os adolescentes não contaram sobre sua sexualidade ou identidade de gênero para a família. Além disso, o adolescente não se sente seguro em conversar sobre a sexualidade por medo do estigma, falta de respeito e problemas relacionados à confidencialidade (Snyder, et al., 2017; Guss, et al., 2019; Fisher, et al., 2018; Kidd, et al., 2020). É importante que o adolescente tenha a oportunidade de revelar sua identidade de gênero e sinta segurança em ser cuidado de maneira afirmativa e acolhedora por profissionais treinados e apoiadores. Por sua vez, o profissional de saúde deve oferecer apoio emocional e prático aos adolescentes de minorias sexuais (Luk, et al., 2020; Clark, et al., 2018; Guss, et al., 2019; Mayer, et al., 2014).

Dentre formas de apoio e suporte a serem ofertados, está a educação em saúde. Os profissionais devem estar capacitados para promover discussões abertas com os jovens; fornecer informações inclusivas com os jovens sobre orientação sexual, identidade de gênero e saúde mental, além de ajuda-los a encontrar uma maneira de conversar com os pais/familiares sobre a temática (Lait, et al., 2019).

Os pais/ adultos significativos devem compreender o papel valorizado que desempenham na vida do adolescente transgênero de forma a facilitar o recebimento de cuidados de saúde, realizar discussões sobre identidade de gênero, saúde juvenil e organizações amigas LGBT / centros de saúde em sua área local, afinal percebe-se que maior conexão com os pais está associado a aumento no recebimento de cuidados em saúde e odontológicos (Taliaferro, et al., 2019).

A rejeição familiar pode levar estes ao isolamento social, expulsão de sua casa, situação financeira e social instável (Scott, et al., 2016; Lindsay, et al., 2019). Assim, quando os pais não conseguirem ofertar apoio, o profissional deve buscar adultos significativos que possam estimular o uso do serviço de saúde e prevenir problemas de saúde mental. Os profissionais devem respeitar e encorajar os relacionamentos que promovem a utilização de serviços de saúde preventivos, particularmente para pessoas transgênero de baixa renda e de minorias raciais / étnicas, uma vez que eles criam um ambiente mais acolhedor e facilitam o reconhecimento da identidade (Van Donge, et al., 2019; Taliaferro, et al., 2019).

Entre os estudos selecionados nessa revisão foi possível identificar que todos tratavam sobre a necessidade de capacitação dos profissionais de saúde em relação ao conhecimento sobre jovens LGBT's, além de discutirem sobre a heterocisnormatividade como um processo social que cria estigmas sociais sobre sexualidade e identidade de gênero, dificultando ações em saúde capazes de contemplar a diversidade em seu todo. Nesse sentido, pode-se definir a importância da prestação de serviços voltados para essa população, identificar as necessidades de adolescentes de minorias sexuais e de gênero e a possibilidade de capacitar profissionais para que possam melhorar a prestação de cuidados primários à saúde (Snyder, et al., 
2016). Durante o preparo dos profissionais, temáticas direcionadas a abordagem da saúde das minorias sexuais e de gênero, bem como o estudo de políticas publicas deveriam ocorrer de forma transversal nos currículos (Matta, et al, 2020).

A capacitação de profissionais deve incluir temáticas como políticas de saúde voltadas para essa população e a abordagem em saúde. Entretanto é importante uma formação mais extensa que vise desconstruir preconceitos enraizados e estimule os profissionais a refletirem sobre as especificidades das minorias sexuais e de gênero. Baseados na compreensão das necessidades de saúde, bem como na literatura, os trabalhadores de saúde devem encorajar mudanças no atendimento em saúde de forma que ele se torne mais inclusivo, deixe de causar constrangimentos e promova um acesso de qualidade ao atender os princípios do SUS (Oliveira, et al., 2021).

O estudo teve como um de seus limites a nacionalidade dos artigos, pois nenhum é brasileiro. Mesmo que em sua maioria os estudos sejam feitos fora do Estado brasileiro, eles auxiliam a problematizar questões vivenciadas por esses adolescentes. Apesar de existir no Brasil a Política de Assistência Integral à saúde da população de Lésbicas, Gays, Bissexuais, Travestis, Transexuais - LGBT e a Política Nacional de Atenção Básica (PNAB), ainda precisamos de mais pesquisas que discutam as necessidades de jovens brasileiros e as intervenções em saúde necessárias a serem implementadas pelos serviços de saúde como ações de promoção em saúde e atividades educativas com vistas a atender as necessidades singulares e a possibilidade de autonomia (Guimarães, et al., 2019).

Outra questão foi relacionada ao fato de que a maioria dos artigos pesquisam adolescentes transgêneros, não sendo encontrado nenhum sobre lésbicas ou bissexuais especificamente, por exemplo. Pensamos que talvez, eles possam ter necessidades específicas. A despeito disso, o estudo conseguiu encontrar intervenções indicadas pela literatura que podem auxiliar os serviços e profissionais a serem mais acolhedores e promover ações que venham ao encontro das reais necessidades dessa população.

É clara a importância dessa pesquisa para a atuação do assistente social já que são profissionais que atuam no atendimento desses adolescentes de minorias sexuais e de gênero que vivenciam diversas expressões da questão social, como a discriminação e violências causadas pela sexualidade e orientação sexual. Sendo ressaltado pela lei 8.662/93 (Código de Ética Profissional) que o assistente social deve realizar seu exercício profissional “(...) sem sofrer discriminação nem discriminar por questões de inserção de classe social, gênero, etnia, religião, nacionalidade, orientação sexual, identidade de gênero, idade e condição física". Torna-se dever do profissional lutar por espaços que sejam acolhedores, incentivando o respeito, a diversidade, a participação de grupos discriminados nos diversos espaços institucionais e o empenho na luta contra preconceitos (Brasil, 2012).

\section{Considerações Finais}

Adolescentes de minorias sexuais e de gêneros perpassam por diversos estigmas e preconceitos sociais até a vida adulta, acarretando em riscos para sua saúde e o afastamento destes de serviços de saúde. Os resultados deste Scoping Review indicam intervenções a serem realizadas no sentido de superar as consequências negativas do estigma e preconceito, além de aproximar os adolescentes transgêneros dos serviços de saúde.

Os estudos indicam melhorias a serem realizadas na acessibilidade e disponibilidade dos serviços como ambientes mais acolhedores prontuários que abordem a diversidade, presença de materiais educativos nas salas de espera, treinamentos com os profissionais de saúde considerando a extensa diversidade que esses adolescentes vivenciam, uso de tecnologias como o telessaúde. $\mathrm{O}$ atendimento deve ser considerar o sigilo, confidencialidade e privacidade, realizadas por profissionais capacitados. 
As políticas de saúde que facilitem a acessibilidade e disponibilidade aos cuidados em saúde, implementação de ações de promoção à saúde, educação para pais e rede social, bem como ações de suporte/apoio estão recomendadas para estimular a presença dos adolescentes no cuidado em saúde.

Percebe-se que o campo de estudo é pouco explorado pela literatura nacional e os estudos são observacionais, ocorrendo uma lacuna em relação a diversidade de atendimento para minorias sexuais e de gênero que deve ser preenchida por estudos futuros. É importante que estudos futuros utilizem metodologias intervencionistas com enfoque no atendimento de necessidades de saúde dessa população. Por outro lado, os artigos trouxeram importantes intervenções que podem ser realizadas para melhorar o atendimento à população/o acesso e até mesmo a promoção da saúde.

\section{Referências}

Belém, J. M., Alves, M. J. H., Pereira, E. V., Moreira, F. T. L. S., Quirino, G. S. \& Albuquerque, G. A. (2018). Atenção à saúde de Lésbicas, Gays, Bissexuais, Travestis e Transexuais na Estratégia Saúde da Família. Rev baiana enferm, 32, 1-13.

Brasil, (2012). Código de ética do/a assistente social. Lei 8.662/93 de regulamentação da profissão. Conselho Federal de Serviço Social.

Brasil, (2013). Política Nacional de Saúde Integral de Lésbicas, Gays, Bissexuais, Travestis e Transexuais. Ministério da Saúde.

Cafferty, R., Desai, B., Alfath, Z., Davey, C. \& Schneider, K. (2020). Adolescent Pride Festival Attendees-Assessing Their Interactions With Primary Care Physicians. J Adolesc Health, 66 (6), 666-671.

Clark, B. A., Veale, J. F., Greyson, D. \& Saewyc E. (2018). Primary care access and foregone care: a survey of transgender adolescents and young adults. Fam Pract. May 23, 35(3), 302-306.

Fisher, C. B., Fried, A. L., Desmond, M., Macapagal, K. \& Mustanski, B. (2018). Perceived Barriers to HIV Prevention Services for Transgender Youth. LGBT Health, 5(6), 350-358.

Gahagan, J. \& Subirana-Malaret, M. (2018). Improving pathways to primary health care among LGBTQ populations and health care providers: key findings from Nova Scotia, Canada. Int J Equity Health,13;17(1), 76.

Goldenberg, T., Jadwin-Cakmak, L., Popoff, E., Reisner, S. L., Campbell, B. A. \& Harper, G. W. (2019). Stigma, Gender Affirmation, and Primary Healthcare Use Among Black Transgender Youth. J Adolesc Health, 65(4), 483-490.

Guimarães, N. A., Marqui, G. D. S., Brum, M. L. B., Vendruscolo, C., Werner \& J. M., Zanatta, E. A. (2019). Relatos de jovens homoafetivos sobre sua trajetória e implicações para a saúde mental. Escola Anna Nery, 23(1), 1-9.

Guss, C. E., Eiduson, R., Khan, A., Dumont, O., Forman, S. F. \& Gordon, A. R. (2020). "It'd Be Great to Have the Options There": A Mixed-Methods Study of Gender Identity Questions on Clinic Forms in a Primary Care Setting. J Adolesc Health, 67(4), 590-596.

Guss, C. E., Woolverton, G. A., Borus, J., Austin, S. B., Reisner, S. L. \& Katz-Wise, S. L. (2019). Transgender Adolescents' Experiences in Primary Care: A Qualitative Study. J Adolesc Health, 65(3), 344-349.

House, H., Gaines, S., \& Hawkins, L. A. (2019). Sexual and Gender Minority Adolescents: Meeting the Needs of Our LGBTQ Patients and Their Families. Clinical Pediatric Emergency Medicine, 20 (1), 9-16.

Luk, J. W., Parker, E. O., Richardson, L. P. \& McCarty, C. A. (2020). Sexual attraction and experiences in the primary care setting: Examining disparities in satisfaction with provider and health self-efficacy. J Adolesc, 81, 96-100.

Matta, T. F., Santos Junior, E. C., Costa, C. M. A. \& Araujo, L. M. (2020). Currículo de saúde e enfermagem LGBT: visão de futuros enfermeiros. Pesquisa, Sociedade e Desenvolvimento. 9(9), e722997855. 10.33448/rsd-v9i9.7855.

Melnyk, B. M. \& Fineout-Overholt, E. (2005). Making the case for evidence-based practice. In: Melnyk B.M., Fineout-Overholt, E. Evidence-based practice in nursing \& healthcare: a guide to best practice. Philadelphia: Lippincot Williams \& Wilkins, 3-24.

Oliveira, B. A. S., Cintra, S. M., Araújo, L. S. A., Santos, J. V. A., Silva, M. E. C., \& Cardoso, G. M. P. (2021). Atenção às pessoas LGBTQIA+ e a não aplicabilidade dos princípios do SUS: Universalidade, Integralidade e Equidade. Pesquisa, Sociedade e Desenvolvimento, 10 (15), e432101523157.

Peters, M. D. J., Godfrey, C., Khalil, H., McInerney, P., Soares, S. C. \& Parker, D., (2017). Scoping reviews. In: Aromataris, E., Munn, Z., editors. Joanna Briggs Institute Reviewer's Manual. The Joanna Briggs Institute.

Snyder, B. K., Burack, G. D. \& Petrova, A. (2017). LGBTQ Youth's Perceptions of Primary Care. Clin Pediatr (Phila), 56(5), 443-450.

Taliaferro, L. A., Harder, B. M., Lampe, N. M., Carter, S. K., Rider, G. N., Eisenberg, M. E., (2019). Social Connectedness Factors that Facilitate Use of Healthcare Services: Comparison of Transgender and Gender Nonconforming and Cisgender Adolescents. J Pediatr, 211, 172-178.

Tricco, A. C., Lillie, E., Zarin, W., Brien, K. K., Colquhoun, H., Levac, D., et al., (2020). PRISMA Extension for Scoping Reviews (PRISMA-ScR): checklist and explanation. Ann Intern Med [Internet], 169(7), 467-73. 
Research, Society and Development, v. 11, n. 2, e36011225766, 2022

(CC BY 4.0) | ISSN 2525-3409 | DOI: http://dx.doi.org/10.33448/rsd-v11i2.25766

Tricco, A. C., Lillie, E., Zarin, W., O'Brien, K., Colquhoun, H., Kastner, M., Levac, D., N. G. C., Sharpe, J. P., Wilson, K., Kenny, M., Warren, R., Wilson, C., Stelfox, H. T., Straus, S. E., (2016). A scoping review on the conduct and reporting of scoping reviews. BMC Med Res Methodol, 9, 16-15.

Van Donge, N., Schvey, N. A., Roberts, T. A., Klein, D. A. (2019). Transgender Dependent Adolescents in the U.S. Military Health Care System: Demographics, Treatments Sought, and Health Care Service Utilization. Mil Med, 184(5-6), 447-454. 